\title{
Optimizing management after spinal cord lesion through integration of individual clinical expertise and best external evidence
}

\author{
JJ Wyndaele, Editor \\ Antwerp University Hospital, Antwerp, Belgium \\ E-mail: spinalcord@uza.be
}

Dear Spinal Cord reader,

The comprehensive management of individuals who have suffered a spinal cord lesion has evolved considerably over the last few decades. The knowledge of what happens in the body, after such a lesion, has increased tremendously and one can expect much more progress to be made. New data may help to optimise the individual's management or help to better understand why traditionally developed techniques are successful. Evidence-based management is high in the requirements. It is about integrating individual clinical expertise and the best external evidence. ${ }^{1}$ These can be acquired through many things; for example, specialisation, feedback, critical evaluation, longitudinal observations, communication and standardisation.

In this issue, several important observations are published. A comprehensive review by BieringSørensen et al. looked into the non-pharmacological treatment and prevention of bone loss after spinal cord injury. No studies conclusively demonstrated an effective intervention, but standing or walking early after a lesion may help retain bone mineral. There also seems to be an effect of electrical stimulation (ES) in this stage. In the chronic phase, weight bearing seems to have little effect, but improvement is seen with longer periods of training, higher frequency or ES stimulus intensity. Impact vibration and pulsed electromagnetic fields did seem to have a positive effect, whereas pulsed ultrasound did not. The authors conclude that bone-mineral should be measured around the knee, the length and intensity of the treatment should be sufficiently long and high and it should commence soon after SCI.

Another review by Pouw et al. shows that because of the limited availability of sensitive prognostic tools to determine the extent of SCI during the acute phase, neurochemical biomarkers of SCI should be evaluated and validated in future clinical trials.

Urodynamics are considered mandatory to guide treatment of the lower urinary tract dysfunction. A study from Vietnam shows that with a cheap one-channel system and sufficient knowledge, good information can be gathered.

In the lower urinary tract, several changes have been demonstrated in both urothelium and afferent nerves after SCI. Pannek et al. demonstrate in patients with suprasacral spinal cord injury, that P2X2 expression seems to be comparable to the expression in patients with idiopathic overactive bladder. Therefore, P2X2 receptors in detrusor tissue may be a future target for the treatment of detrusor overactivity.

One of the important complications after SCI is urinary tract infection. In a pilot study, Prasad et al. showed, that E. coli 83972-coated urinary catheters were a viable way of achieving bladder colonization with this potentially protective strain in persons practising intermittent catheterisation. This correlated with a decrease in infection rate.

Hetz et al. have, by identifying common activities of daily living (ADL) performed by individuals with SCI, begun to provide direction for developing strategies to optimize ADL participation. They propose that future research should examine fitness as a way to help individuals with SCI optimize their ADL participation.

More, equally interesting, studies are published in this issue. All of which help to improve the quality of management.

\section{References}

1 Sackett DL, Rosenberg WM, Gray JA, Haynes RB, Richardson WS. Evidence based medicine: what it is and what it isn't. BMJ 1996; 312: 71-72.

Spinal Cord (2009) 47, 507; doi:10.1038/sc.2009.80 